DR. ULVI KAHRAMAN GURSOY (Orcid ID : 0000-0002-1225-5751)

PROF. ANNA LIISA LIISA SUOMINEN (Orcid ID : 0000-0002-8543-0055)

Article type : Original Article

\title{
Molecular Forms and Fragments of Salivary MMP-8 in Relation to Periodontitis
}

Ulvi K. Gürsoy ${ }^{1}$, Eija Könönen ${ }^{1,2}$, Taina Tervahartiala ${ }^{3}$, Mervi Gürsoy ${ }^{1}$, Jari Pitkänen ${ }^{3}$, Paula Torvi $^{3}$, Anna Liisa Suominen ${ }^{4,5,6}$, Pirkko Pussinen $^{3}$, Timo Sorsa $^{3,7}$

1 Periodontology, Institute of Dentistry, University of Turku, Turku, Finland

2 Oral Health Care, Welfare Division, City of Turku, Turku, Finland

3 Department of Oral and Maxillofacial Disease, University of Helsinki, Helsinki University Hospital, Helsinki, Finland

4 Institute of Dentistry, University of Eastern Finland, Kuopio, Finland

5 Health Monitoring Unit, National Institute for Health and Welfare, Helsinki, Finland

6 Department of Oral and Maxillofacial Diseases, Kuopio University Hospital, Kuopio, Finland

7 Periodontology, Department of Dental Medicine, Karolinska Institutet, Huddinge, Sweden

Running Title: Salivary MMP-8 in periodontitis

Key words: Collagenase; Neutrophil; Periodontal Disease; Saliva

This article has been accepted for publication and undergone full peer review but has not been through the copyediting, typesetting, pagination and proofreading process, which may lead to differences between this version and the Version of Record. Please cite this article as doi: $10.1111 /$ jcpe. 13024

This article is protected by copyright. All rights reserved. 


\section{Corresponding Author:}

Ulvi Kahraman Gursoy, Institute of Dentistry, University of Turku, Lemminkäisenkatu 2, 20520 Turku, Finland, Tel: +358 2 3338335, E-mail: ulvi.gursoy@utu.fi

\section{Conflict of Interest and Source of Founding}

This study was supported by grants from the Helsinki University Hospital Research Foundation (TYH2016251, TYH 2018229, TYH2017251, Y1014SL017, Y1014SL018) Helsinki, Finland, Karolinska Institutet, Stockholm, Sweden, and Academy of Finland. Prof. Timo Sorsa is an inventor of US-patents 5652223, 5736341, 5866432, and 6143476.

\section{Clinical Relevance}

Scientific rationale for study: Matrix metalloproteinase (MMP)-8 is the major collagenase in gingival crevicular fluid and its levels in gingival tissues and oral fluids are significantly elevated in periodontitis. It is secreted in its inactive pro-form and gets activated by auto- or bacterial degradation.

Principal findings: The fragments of salivary MMP-8 were elevated in both localized (early) and generalized (advanced) forms of periodontitis. Treponema denticola chymotrypsin-like protease can activate the inactive pro-MMP-8 and form MMP-8fragments.

This article is protected by copyright. All rights reserved. 
Practical implications: Future diagnostic adjunctive quantitative MMP-8 point-ofcare/chair-side technologies need to utilize MMP-8 antibodies selective for MMP-8 activation cascade and fragments.

\begin{abstract}
Aim: To investigate the molecular forms of salivary matrix metalloproteinase (MMP)-8 in relation to periodontitis.
\end{abstract}

Materials and Methods: Molecular forms, degree of activation, and fragmentation of neutrophilic and mesenchymal type MMP-8 isoforms were analyzed from salivary samples of 81 subjects with generalized periodontitis, 63 subjects with localized periodontitis, and 79 subjects without pocket teeth, by using western-immunoblots with computer quantitation. In addition, human recombinant proMMP-8 was in vitro activated by Treponema denticola chymotrypsin-like protease (Td-CTLP), sodium hypochlorite ( $\mathrm{NaOCl}, 1 \mathrm{mM}$, oxidant), or amino phenyl mercuric acetate (APMA, $1 \mathrm{mM}$ ).

Results: In saliva of periodontitis-affected individuals, MMP-8 is found in multiple forms, i.e., complexes, active and pro-forms of neutrophilic and mesenchymal-type MMP-8, and especially 20-27 $\mathrm{kDa}$ fragments. The quantity of these fragments was elevated in both localized and generalized forms of periodontitis. Moreover, the tested activators (Td-CTLP, $\mathrm{NaOCl}$, and APMA) activated inactive proMMP-8, resulting in fragments of 20-27 kDa, in vitro, and salivary concentrations of $T$. denticola correlated significantly with salivary levels of fragmented MMP-8.

This article is protected by copyright. All rights reserved. 
Conclusion: The present results indicate that during the development and progression of periodontitis, MMP-8 appears as activated and fragmented, and treponemal proteases most likely play role in this cascade.

\section{Introduction}

In health, matrix metalloproteinases (MMPs) function in tissue development, homeostasis and remodeling, cell migration and wound healing, immune cell activation, and antimicrobial defense, and their activities are regulated by tissue inhibitors of matrix metalloproteinases. Their pathologically elevated expressions, however, may lead to irreversible tissue destruction, as in tumor angiogenesis and metastasis, vascular diseases, and periodontitis (Rohani and Parks, 2015).

In the oral cavity, various cell types express and secrete MMPs. Neutrophils, which form a continuous barrier in the gingival crevice between bacterial biofilm and the epithelium, degranulate MMP-8 (collagenase-2 or neutrophilic collagenase), being of major importance in etiopathogenesis of periodontal disease (Alfakry et al., 2016). In periodontitis, MMP-8 is the major collagenase in periodontal tissues and over $90 \%$ of the collagenase activity in gingival crevicular fluid (GCF), saliva, and mouthrinse originates from MMP-8 (Sorsa et al., 2016). While MMP-8 is one of the most important degradative enzymes in pathogenesis of periodontal disease at pathologically elevated levels, its total absence disrupts host-defensive response, which associates with progression of periodontitis and systemic inflammation, as demonstrated in a MMP-8 knockout-mice model (Kuula et al., 2009). Elevated levels of activated MMP-8 in oral fluids have been proposed as diagnostic markers of periodontitis (Gursoy et al., 2010, 2018; Ebersole et al., 2015; Sorsa et al., 2016). 
By using immunodetection methods, MMP-8 can be found in several molecular forms in GCF and peri-implant sulcus fluid; these include complexes at sizes of $>110 \mathrm{kDa}$, latent (pro) and active forms of neutrophilic MMP-8 at 70-80 kDa, mesenchymal-type collagenase at 50$65 \mathrm{kDa}$ with latent (pro) and active forms, and fragments at 20-30 kDa (Hasty et al., 1986; Ding et al., 1997; Palosaari et al., 2000; Kiili et al., 2002; Reynaud af Geijersstam et al., 2005). It has been shown that Treponema denticola, a major periodontitis-associated bacterium, can activate proMMP-2 with its chymotrypsin-like protease (Miao et al., 2011). Active neutrophilic $55 \mathrm{kDa}$ MMP-8 is considered one major form in periodontitis (Romanelli et a., 1999; Sorsa et al., 2010). Detecting high levels of active MMP-8 in saliva has a strong diagnostic ability in comparison to methods that detect total MMP-8 levels (Gursoy et al., 2010). Although molecular forms of MMP-8 have been demonstrated in GCF and periimplant sulcus fluid (Kiili et al., 2002; Kivelä-Rajamäki et al., 2003; Sorsa et al., 2010), to our knowledge, relationships of different molecular forms of salivary MMP-8 fractions to periodontitis have not been studied yet. Moreover, the activation of proMMP-8 by $T$. denticola chymotrypsin-like protease (Td-CTLP) has not been demonstrated before. According to our hypothesis, in periodontitis, secreted MMP-8 is subjected to auto- or bacterial degradation, and MMP-8 fragments become detectable as early diagnostic markers of active disease and disease severity. With this background, the aim was to examine the molecular forms of MMP-8 in saliva, and the degree of activation and fragmentation of MMP-8 in relation to periodontitis.

This article is protected by copyright. All rights reserved. 


\section{Material and Methods}

\section{Activation of human recombinant proMMP-8 by Td-CTLP, sodium hypochlorite (NaOCl), and Amino Phenyl Mercuric Acetate (APMA)}

Purified PMN MMP-8 was prepared as described by Hanemaaijer et al. (1997). Human proMMP-8 $(20 \mu \mathrm{g})$ was treated with Td-CTLP, as described by Sorsa et al. (1992), $1 \mathrm{mM}$ $\mathrm{NaOCl}$ (Pharmacy of University of Helsinki, Helsinki, Finland), and 1 mM APMA (Sigma, St Luis, MO, USA) and the activations were monitored by western-immunoblotting (Saari et al., 1990; Ding et al., 1996).

\section{Saliva samples}

The paraffin-stimulated saliva samples came from participants of the Southern Finland district of the national Finnish Health 2000 survey (BRIF8901) and the clinical data from oral and radiographic examinations of the survey participants are described in the report of the National Public Health Institute (http://www.terveys2000.fi/indexe.html). All participants gave written informed consent, and study protocols were approved by the Ethical Committee for Epidemiology and Public Health of the Hospital District of Helsinki and Uusimaa, Finland (BRIF8901, 407/E3/2000/terveys2000).

Digital panoramic radiographs were used to determine the alveolar bone loss (Gursoy et al., 2013). Radiographs were taken with a dental panoramic x-ray machine (imaging values between 58-68 kV and 4-10 mA, PM 2002 CC proline apparatus; Planmeca, Helsinki, Finland) and bone levels of each tooth (mesial and distal surfaces and on the furcation areas of molar teeth) were analyzed by a specialist in oral radiology using an inbuilt measuring tool of Dimaxis software (Planmeca). The Advisory Board for Radiation Safety approved 
radiographical examination protocols and the Radiation and Nuclear Safety Authority and the Ethics Committees for Human Studies of the National Public Health Institute and the Institute of Epidemiology and National Health, Finland granted the safety licenses.

The samples were transported in carbonic acid ice and immediately frozen at $-70^{\circ} \mathrm{C}$ until further use. When the saliva samples were thawed for the first time, they were centrifuged at $9300 \mathrm{~g}$ for $5 \mathrm{~min}$, and the supernatants were used for the biochemical assays.

A total of 223 saliva samples, collected from 81 subjects with generalized periodontitis (GP) [14 or more teeth with $\geq 4 \mathrm{~mm}$ probing pocket depth (PPD)], 63 subjects with localized periodontitis (LP with two subgroups; LP1: 2 teeth with PPD of $\geq 4 \mathrm{~mm}$, and LP2: 7 teeth with PPD of $\geq 4 \mathrm{~mm}$ ), and 79 subjects without pocket teeth (control), were included in the present study. Mean bone loss levels were $33.3 \pm 41.4 \mathrm{~mm}$ for GP, $11.1 \pm 12.5 \mathrm{~mm}$ for LP1, 18.3 \pm 26.2 for LP2, and 3.5 $\pm 5.4 \mathrm{~mm}$ for the control group (Gursoy et al., 2013). All patients in the GP and LP groups showed bone loss and the patient was used as the unit of analysis. All Health 2000 survey subjects who fit into above mentioned groups were included in the study. Mean age (with minimum-maximum values) of the participants in four periodontal groups were as follows: 49.6 (41-60) years for GP, 48.0 (40-60) years for LP1, 49.4 (41-59) years for LP2, and 47.9 (40-60) years for the control group. Percentages of smokers were $56.6 \%$ for GP, $25.0 \%$ for LP1, $34.5 \%$ for LP2, and $23.5 \%$ for the control group. Male percentage was $60.2 \%$ for the GP, $41.7 \%$ for LP1, $41.4 \%$ for LP2, and $35.8 \%$ for the control group.

This article is protected by copyright. All rights reserved. 


\section{Immunoblotting of salivary MMP-8}

All samples were analyzed together and the laboratory staff was unaware of the study groups in which the samples belong to. For detecting the molecular forms of salivary MMP-8, a modified method of the enhanced chemiluminescence (ECL) Western immunoblotting kit (GE Healthcare, Amersham, UK) was used according to the protocol recommended by the manufacturer (Lauhio et al., 1994; Hanemaajier et al., 1997). Briefly, $14 \mu \mathrm{l}$ of saliva were mixed with Laemmli's buffer without the reducing reagent and heated for $5 \mathrm{~min}$, and protein separations were performed with $11 \%$ sodium dodecyl sulphate (SDS)-polyacrylamide gels. After electrophoresis, the proteins were electrotransferred onto nitrocellulose membranes (Protran, Whatman GmbH, Dassel, Germany), which were incubated overnight with a primary polyclonal antibody, anti-MMP-8 (Lauhio et al., 1994; Hanemaaijer et al., 1997). Target detection was performed according to the manufacturer's protocol. The quantitation of immunoblots was performed by using the GS-700 Imaging Densitometer Scanner and the Bio-Rad Quantity One program (Bio-Rad, Hercules, CA, USA).

\section{Detection of Treponema denticola}

Salivary $T$. denticola levels were available for the control and GP groups from our previous study (Hyvärinen et al., 2009). The analysis of $T$. denticola was performed using a single copy gene-based real-time PCR (Hyvärinen et al., 2009). The results are expressed as concentrations using genomes per milliliter (GE/ml).

This article is protected by copyright. All rights reserved. 


\section{Follow-up Study}

A part of the participants of the national Finnish Health 2000 survey (BRIF8901), who were at the age of 18 or older in 2000, were invited to a follow-up study, Health 2011 survey. Of the 223 participants of Health 2000 survey, whose saliva samples were analyzed in the present study, 120 participated to clinical oral health examination in the Health 2011 survey as well. A detailed description of the Health 2011 survey and its clinical oral examinations can be found elsewhere (Suominen et al., 2018). Study protocols were approved by the Ethical Committee for Epidemiology and Public Health of the Hospital District of Helsinki and Uusimaa, Finland and all participants gave informed consent (45/13/03/00/11 for the Health 2011 survey).

To define the predictive capabilities of each molecular form of MMP-8 present in saliva, salivary levels of MMP-8 forms were first divided into tertiles and then the participants were grouped according their tertile scores. Number of teeth with deepened pockets (PPD $\geq 6 \mathrm{~mm}$ ) in 2011 was taken as outcome variable.

\section{Statistical analyses}

All statistical analyses were performed with the SPSS statistical program (version 23.0; IBM Armonk, New York, USA). The data distributions of MMP-8 fraction levels were skewed, therefore, non-parametric tests were applied. The Kruskal-Wallis $\mathrm{H}$ (for multiple comparisons) and Mann-Whitney U tests were used for comparing the levels of the molecular forms of MMP-8 between the four periodontal groups. The Spearman test was used to analyze the correlations between different forms of MMP-8 and salivary T. denticola levels. A statistical significance was defined as a p-value $<0.05$.

This article is protected by copyright. All rights reserved. 


\section{Results}

Purified MMP-8 was in part autofragmented and self-activated. Human 70-80 kDa proMMP8 was converted to its $50-60 \mathrm{kDa}$ active forms by tested Td-CTLP, HOCl, and APMA, and further induced to the fragmented lower molecular size of 20-27 kDa fragments (Fig. 1 A-C).

The molecular forms, activations, and fragments of salivary MMP-8 are given in Figure 2. The relative levels of MMP-8 complexes at $>110 \mathrm{kDa}$ were higher in LP2 group than in controls $(\mathrm{p}=0.032)$. No difference was observed in pro-forms of neutrophilic MMP-8. The active form of neutrophilic MMP-8 was lowest in LP1 group, in comparison to the control $(\mathrm{p}=0.033)$ and GP $(\mathrm{p}=0.002)$ groups. Neither the pro-form nor the active form of mesenchymal MMP-8 differed between the periodontal groups. The 20-27 kDa fragments of MMP-8 were elevated in LP1 $(\mathrm{p}<0.001)$, LP2 $(\mathrm{p}<0.001)$, and GP $(\mathrm{p}<0.001)$ groups, when compared with the control group (Figure 3).

Salivary levels of $T$. denticola were $1.64 \times 10^{4} \mathrm{GE} / \mathrm{ml}$ for the GP group and $5.64 \times 10^{3} \mathrm{GE} / \mathrm{ml}$ for the controls. There were significant correlations between fragments of MMP-8 and $T$. denticola (Spearman's rho=189, $\mathrm{p}=0.016$ ), and between the active form of mesenchymal MMP-8 and T. denticola (Spearman's rho=207, p=0.008).

Figure 4 presents the ROC curves for discriminating the periodontitis and control groups. The 20-27 kDa fragments of MMP-8 were able to distinguish the subjects with and without periodontitis and provided AUCs larger than $0.7(\mathrm{p}<0.001)$.

GP patients in the highest tertiles of fragmented $(\mathrm{p}=0.021)$ form of MMP-8 had a higher number of teeth with deepened pockets (PPD $\geq 6 \mathrm{~mm}$ ) in the follow-up Health 2011 study, in comparison to those who were in the lowest tertiles of these molecular forms.

This article is protected by copyright. All rights reserved. 


\section{Discussion}

In the present study, we demonstrated that MMP-8 present in saliva can be observed in complex, active and pro neutrophilic, and active and pro mesenchymal forms, as well as in low molecular weight $(20-27 \mathrm{kDa})$ fragments. Among these molecular forms, the fragments of salivary MMP-8 were elevated in both localized (early) and generalized (advanced) forms of periodontitis.

This is the first study to characterize the molecular forms and fragmentation of salivary MMP-8 in periodontitis. A previous study, dealing with molecular forms and levels of MMP8 in GCF, demonstrated increases in active forms of neutrophilic and mesenchymal MMP-8 during periodontitis, whereas fragments of MMP-8 were barely detectable (Kiili et al., 2002). In another study, using peri-implantitis sulcus fluid, all six forms of MMP-8 were found at higher levels in peri-implant mucositis and peri-implantitis cases in comparison to those in healthy controls (Kivelä-Rajamäki et al., 2003). The salivary profile of MMP-8 in periodontitis seems to differ, at least partly, from its profile in GCF during periodontitis and in peri-implant sulcular fluid during peri-implantitis.

Neutrophilic MMP-8 is secreted, or degranulated, as a $75 \mathrm{kDa}$ molecule in pro-form and is reduced to its active form at $57 \mathrm{kDa}$ (Hasty et al., 2006). The 20-27 kDa fragments of MMP-8 are degradation end-products of $57 \mathrm{kDa}$ neutrophilic MMP-8 activation and are without collagenase activity (Hasty et al., 1986). Release, activation, and fragmentation of MMP-8 upon degranulation from neutrophils is regulated by bacteria (Reynaud af Geijersstam et al., 2005), especially by periodontitis-associated pathogens (Ding et al., 1996; 1997). Bacterial enzymes convert pro neutrophilic MMP-8 to its active form and matrilysin-1 (MMP-7) is responsible for its proteolytical cleavage (Sorsa et al., 1992; Dozier et al., 2006). MMP-8 can also be activated by oxidative mechanisms without having a reduction in its molecular weight 
(Saari et al., 1990). MMP-8 fragments, on the other hand, are end-products of auto- and bacterial degradation of degranulated 57 kDa MMP-8 (Reynaud af Geijersstam et al., 2005; Hasty et al., 2006). The degradation of $57 \mathrm{kDa}$ MMP-8 by $T$. denticola was demonstrated in this study as well. Moreover, salivary levels of fragmented MMP-8 correlated significantly with salivary $T$. denticola concentrations. In the present study, only low levels of active neutrophilic MMP-8 were found in saliva of LP1 group in comparison to the generalized periodontitis and control groups. This might be explained by the bacterial degradation of MMP-8 in initial periodontitis (here: in subjects with 2 pocket teeth). The released MMP-8, which is at low levels during initial periodontitis, can be subjected to bacterial proteases and get degraded (Sorsa et al., 1992; Nieminen et al., 2017). Active neutrophilic MMP-8 levels of LP2 and GP groups were higher than those of LP1, but at the same time similar to that of controls. This outcome might be explained by the simultaneous elevations of neutrophilic MMP-8 and bacterial proteases with the extension of the disease. In the present study, the gradual extension of periodontal disease from localized to generalized disease leads to consistently increased levels of low molecular size (20-27 kDa) fragments of MMP-8 and the ROC curve analysis results supported this statement. Our findings on salivary MMP-8 fragments suggest that their levels increase with the progression of periodontitis and stay at elevated levels during extension of the disease. Indeed, it seems that full-size pro-form MMP8 is ready for a potential activation and fragmentation upon periodontal disease initiation and progression, and the occurrence of elevated levels of MMP-8 fragments is an outcome of this extended MMP-8 activation. In fact, corresponding MMP-8 activation and fragmentation cascades have been described during neutrophilic degranulation in vitro (Hasty et al., 1986; Reynaud af Geijersstam et al., 2005) and in vivo in other body fluids and secretions, such as diabetic nephropathic urine, glaucoma-affected tear fluid, and bronchiectasis-affected bronchoalveolar lavage (Prikk et al., 2002; Määttä et al., 2006; Lauhio et al., 2008). The most 
common methods to detect MMP-8 in body fluids are the enzyme-linked immunosorbent assay and time-resolved immunofluorometric assay (IFMA). These two methods measure total MMP-8 levels, or latent and active forms of MMP-8, alone or together (Sorsa et al., 2016; Sorsa et al., 2017). The only method to analyze molecular forms and/or fragments of MMP-8 is the computer-scanned western-immunoblots (Kiili et al., 2003; Hernandez et al., 2010; Buduneli et al., 2011). The computer-quantitated western-immuoblots and IFMA have been demonstrated to correlate significantly with each other, indicating that both immunotechniques are suitable for oral fluid MMP-8 analyses (Buduneli et al., 2011). The ROC curve analysis obtained by computer-scanned immunoblots provided AUC scores of $>0.7$ for $20-27 \mathrm{kDa}$ salivary MMP-8 fragments, which corresponds to those previously obtained by MMP-8 IFMA analysis (Gursoy et al., 2010; Mauramo et al., 2017).

In the present study, no clear differences were found in the levels of pro and active forms of mesenchymal MMP-8 between the control and periodontitis groups. The main source of mesenchymal MMP-8 in saliva is fibroblasts, but other cells (odontoblasts) can secrete this MMP-8 isoform as well (Palosaari et al., 2000). Fibroblasts take part in tissue regeneration by producing new collagen and destroying the old one; fibroblastic MMP-8, together with MMP-1 and -13, are the only collagenases that can cleave native type I collagen (Krane 1995). Bacterial infection connected to periodontitis affects immune cells and resident cells in opposite directions. While infection induces neutrophilic chemotaxis and release or selective degranulation of proteases in an uncontrolled manner (Scott and Krauss 2012), bacterial lipopolysaccharide induces apoptosis of fibroblasts (Alikhani et al., 2003). Increase in apoptotic behavior of fibroblasts may explain why fibroblastic (mesenchymal) MMP-8 did not differ between the periodontal groups in our study. Furthermore, upon activation, this isoform of MMP-8 can be fragmented into low molecular weight fragments (Hanemaaijer et al., 1997).

This article is protected by copyright. All rights reserved. 
Based on the present findings, generalized periodontitis patients with elevated fragmented forms of salivary MMP-8 have deepened probing pocket depths after 10 years. To our knowledge, this is one of the first studies to demonstrate the predictive capability of salivary biomarkers in a longitudinal design over 10 years.

In conclusion, fragments of MMP-8 resulting from its activation become detectable in saliva at initial phases of periodontitis and can be considered a possible biomarker of periodontitis. Future diagnostic adjunctive quantitative MMP-8 point-of-care/chair-side technologies need to utilize MMP-8 antibodies selective for MMP-8 activation cascade and fragments.

\section{References}

Alfakry, H., Malle, E., Koyani, C.N., Pussinen, P.J., \& Sorsa, T. (2016). Neutrophil proteolytic activation cascades: a possible mechanistic link between chronic periodontitis and coronary heart disease. Innate Immunity, 22, 85-99.

Alikhani, M., Alikhani, Z., He, H., Liu, R., Popek, B.I., \& Graves, D.T. (2003) Lipopolysaccharides indirectly stimulate apoptosis and global induction of apoptotic genes in fibroblasts. The Journal of Biological Chemistry, 278, 52901-52908.

Buduneli, E., Mäntylä, P., Emingil, G., Tervahartiala, T., Pussinen, P., Barış, N., Akı1lı, A., Atilla, G., \& Sorsa, T. (2011). Acute myocardial infarction is reflected in salivary matrix metalloproteinase-8 activation level. Journal of Periodontology, 82, 716-725.

This article is protected by copyright. All rights reserved. 
Ding, Y., Uitto, V.J., Haapasalo, M., Lounatmaa, K., Konttinen, Y.T., Salo, T., Grenier, D., \& Sorsa, T. (1996). Membrane components of Treponema denticola trigger proteinase release from human polymorphonuclear leukocytes. Journal of Dental Research, 75, 1986-1993.

Ding, Y., Haapasalo, M., Kerosuo, E., Lounatmaa, K., Kotiranta, A., \& Sorsa, T. (1997). Release and activation of human neutrophil matrix metallo- and serine proteinases during phagocytosis of Fusobacterium nucleatum, Porphyromonas gingivalis and Treponema denticola. Journal of Clinical Periodontology, 24, 237-248.

Dozier, S., Escobar, G.P., \& Lindsey, M.L. (2006) Matrix metalloproteinase (MMP)-7 activates MMP-8 but not MMP-13. Medicinal Chemistry, 2, 523-526.

Ebersole, J.L., Nagarajan, R., Akers, D., \& Miller, C.S. (2015). Targeted salivary biomarkers for discrimination of periodontal health and disease(s). Frontiers in Cellular Infection and Microbiology, 5, 62 .

Gursoy, U.K., Könönen, E., Pradhan-Palikhe, P., Tervahartiala, T., Pussinen, P.J., SuominenTaipale, L., \& Sorsa, T. (2010). Salivary MMP-8, TIMP-1, and ICTP as markers of advanced periodontitis. Journal of Clinical Periodontology, 37, 487-493.

Gursoy, U.K., Könönen, E., Huumonen, S., Tervahartiala, T., Pussinen, P.J., Suominen, A.L. \& Sorsa, T. (2013). Salivary type I collagen degradation end-products and related matrix metalloproteinases in periodontitis. Journal of Clinical Periodontology, 40, 18-25.

Gürsoy, U.K., Pussinen, P.J., Salomaa, V., Syrjäläinen, S. \& Könönen, E. (2018). Cumulative use of salivary markers with an adaptive design improves detection of periodontal disease over fixed biomarker thresholds. Acta Odontologica Scandinavica, 20, 1-4 [Epub ahead of print].

This article is protected by copyright. All rights reserved. 
Hanemaaijer, R., Sorsa, T., Konttinen, Y.T., Ding, Y., Sutinen, M., Visser, H., van Hinsbergh, V.W., Helaakoski, T., Kainulainen, T., Rönkä, H., Tschesche, H., \& Salo, T. (1997). Matrix metalloproteinase-8 is expressed in rheumatoid synovial fibroblasts and endothelial cells. Regulation by tumor necrosis factor-alpha and doxycycline. The Journal of Biological Chemistry, 272, 31504-31509.

Hasty, K.A., Hibbs, M.S., Kang, A.H., \& Mainardi, C.L. (1986). Secreted forms of human neutrophil collagenase. The Journal of Biological Chemistry, 261, 5645-5650.

Hyvärinen, K., Laitinen, S., Paju, S., Hakala, A., Suominen-Taipale, L., Skurnik, M., Könönen, E., \& Pussinen, P.J. (2009). Detection and quantification of five major periodontal pathogens by single copy gene-based real-time PCR. Innate Immunity, 15, 195-204.

Kiili, M., Cox, S.W., Chen, H.Y., Wahlgren, J., Maisi, P., Eley, B.M., Salo, T. \& Sorsa, T. (2002). Collagenase-2 (MMP-8) and collagenase-3 (MMP-13) in adult periodontitis: molecular forms and levels in gingival crevicular fluid and immunolocalisation in gingival tissue. Journal of Clinical Periodontology, 29, 224-232.

Kivelä-Rajamäki, M., Maisi, P., Srinivas, R., Tervahartiala, T., Teronen, O., Husa, V., Salo, T., \& Sorsa, T. (2003). Levels and molecular forms of MMP-7 (matrilysin-1) and MMP-8 (collagenase-2) in diseased human peri-implant sulcular fluid. Journal of Periodontal Research, 38, 583-590.

Lauhio, A., Salo, T., Ding, Y., Konttinen, Y.T., Nordström, D., Tschesche, H., Lähdevirta, J., Golub, L.M., \& Sorsa, T. (1994). In vivo inhibition of human neutrophil collagenase (MMP8) activity during long-term combination therapy of doxycycline and non-steroidal antiinflammatory drugs (NSAID) in acute reactive arthritis. Clinial and Experimental Immunology, 98, 21-28.

This article is protected by copyright. All rights reserved. 
Lauhio, A., Sorsa, T., Srinivas, R., Stenman, M., Tervahartiala, T., Stenman, U.H., Grönhagen-Riska, C., \& Honkanen, E. (2008). Urinary matrix metalloproteinase -8, -9, -14 and their regulators (TRY-1, TRY-2, TATI) in patients with diabetic nephropathy. Annals of Medicine, 40, 312-320.

Mauramo, M., Ramseier, A.M., Mauramo, E., Buser, A., Tervahartiala, T., Sorsa, T., \& Waltimo, T. (2017). Associations of oral fluid MMP-8 with periodontitis in Swiss adult subjects. Oral Diseases, Sep 10. [Epub ahead of print], doi: 10.1111/odi.12769.

Miao, D., Fenno, J.C., Timm, J.C., Joo, N.E., \& Kapila, Y.L. (2011). The Treponema denticola chymotrypsin-like protease dentilisin induces matrix metalloproteinase-2dependent fibronectin fragmentation in periodontal ligament cells. Infection Immunity, 79, 806-811.

Määttä, M., Tervahartiala, T., Vesti, E., Airaksinen, J., \& Sorsa, T. (2006). Levels and activation of matrix metalloproteinases in aqueous humor are elevated in uveitis-related secondary glaucoma. Journal of Glaucoma, 15, 229-237.

Nieminen, M.T., Listyarifah, D., Hagström, J., Haglund, C., Grenier, D., Nordström, D., Uitto, V.J., Hernandez, M., Yucel-Lindberg, T., Tervahartiala, T., Ainola, M., \& Sorsa, T. Treponema denticola chymotrypsin-like proteinase may contribute to orodigestive carcinogenesis through immunomodulation. British Journal of Cancer, 2017 [In press]

Palosaari, H., Wahlgren, J., Larmas, M., Rönkä, H., Sorsa, T., Salo, T., \& Tjäderhane, L. (2000). The expression of MMP-8 in human odontoblasts and dental pulp cells is downregulated by TGF-beta1. Journal of Dental Research, 79, 77-84.

This article is protected by copyright. All rights reserved. 
Prikk, K., Maisi, P., Pirilä, E., Reintam, M.A., Salo, T., Sorsa, T., \& Sepper, R. (2002). Airway obstruction correlates with collagenase-2 (MMP-8) expression and activation in bronchial asthma. Laboratory Investigation, 82, 1535-1545.

Reynaud af Geijersstam, A., Sorsa, T., Stackelberg, S., Tervahartiala, T., \& Haapasalo, M. (2005). Effect of E. faecalis on the release of serine proteases elastase and cathepsin G, and collagenase-2 (MMP-8) by human polymorphonuclear leukocytes (PMNs). International Endodontic Journal, 38, 667-677.

Rohani, M.G. \& Parks, W.C. (2015). Matrix remodeling by MMPs during wound repair. Matrix Biology, 44-46, 113-121.

Romanelli, R., Mancini, S., Laschinger, C., Overall, C.M., Sodek, J., \& McCulloch, C.A. (1999). Activation of neutrophil collagenase in periodontitis. Infection and Immunity, 67, 2319-2326.

Saari, H., Suomalainen, K., Lindy, O., Konttinen, Y.T., \& Sorsa, T. (1990). Activation of latent human neutrophil collagenase by reactive oxygen species and serine proteases. Biochemical and Biophysical Research Communications, 171, 979-987.

Scott, D.A. \& Krauss, J. (2012). Neutrophils in periodontal inflammation. Frontiers of Oral Biology, 15, 56-83.

Sorsa, T., Ingman, T., Suomalainen, K., Haapasalo, M., Konttinen, Y.T., Lindy, O., Saari, H., \& Uitto, V.J. (1992). Identification of proteases from periodontopathogenic bacteria as activators of latent human neutrophil and fibroblast-type interstitial collagenases. Infection and Immunity, 60, 4491-4495.

This article is protected by copyright. All rights reserved. 
Sorsa, T., Hernández, M., Leppilahti, J., Munjal, S., Netuschil, L., \& Mäntylä, P. (2010). Detection of gingival crevicular fluid MMP-8 levels with different laboratory and chair-side methods. Oral Diseases, 16, 39-45.

Sorsa, T., Gursoy, U.K., Nwhator, S., Hernandez, M., Tervahartiala, T., Leppilahti, J., Gursoy, M., Könönen, E., Emingil, G., Pussinen, P.J., \& Mäntylä, P. (2016). Analysis of matrix metalloproteinases, especially MMP-8, in gingival creviclular fluid, mouthrinse and saliva for monitoring periodontal diseases. Periodontology 2000, 70, 142-163.

Sorsa, T., Gieselmann, D., Arweiler, N.B., \& Hernández, M. (2017). A quantitative point-ofcare test for periodontal and dental peri-implant diseases. Nature Reviews. Disease Primers, 3, 17069.

Suominen, A.L., Varsio, S., Helminen, S., Nordblad, A., Lahti, S. \& Knuuttila, M. (2018) Dental and periodontal health in Finnish adults in 2000 and 2011. Acta Odontologica Scandinavica, 76, 305-313.

\section{Figure legends}

Figure 1: In vitro activation and fragmentation of human proMMP-8 by Td-CTLP, NaOCl and APMA. The $85 \mathrm{kDa}$ human recombinant proMMP-8 was incubated with Treponema denticola chymotrypsin-like protease (Td-CTLP) for indicated time periods (Fig 1A), oxidant $1 \mathrm{mM} \mathrm{NaOCl}$ (Fig 1B) and organomercurial $1 \mathrm{mM}$ APMA (Fig 1C), and these activations and related fragmentations were monitored by western-immunoblotting. Treponemal protease, oxidant NaOCL, and organomercurial APMA time-dependently activated and fragmented human proMMP-8 (Figs 1A-C).

This article is protected by copyright. All rights reserved. 
Figure 2: Western-immunoblot analysis of salivary samples studied for molecular forms, degree of activation and related fragmentation of MMP-8. Lanes 1-2: control - ; lanes 3-4: LP1; lanes 5-6: LP2; lanes 7-8: GP. Lane 9 indicates neutrophilic (PMN) proMMP-8 (pMMP-8), active PMN MMP-8 (aMMP-8) and fragments (frag.)

Figure 3: Salivary levels of molecular forms and fragments of MMP-8 in subjects with generalized periodontitis [14 or more teeth with $\geq 4 \mathrm{~mm}$ probing pocket depth (PPD)], localized periodontitis (LP1: 2 teeth with $\geq 4 \mathrm{~mm}$ PPD and LP2: 7 teeth with $\geq 4 \mathrm{~mm}$ PPD) and in subjects without pocket teeth (control). Significant differences between the groups were given as connecter lines and p-values.

Figure 4: Receiver operating characteristics (ROC) analysis of each salivary form and fragment of MMP-8, in regard to periodontal disease. Area under the curve (AUC), 95\% confidence intervals, and p-values were given by the ROC curves.

Figure 5: After categorizing the Health 2000 survey participants according to their MMP-8 levels in different molecular forms, their number of teeth with $\geq 6 \mathrm{~mm}$ probing pocket depth (PPD) in 2011 are given as box plots. Only fragmented MMP-8 gave significant differences within the GP group. Statistical differences are marked with connector lines and p-values.

This article is protected by copyright. All rights reserved. 

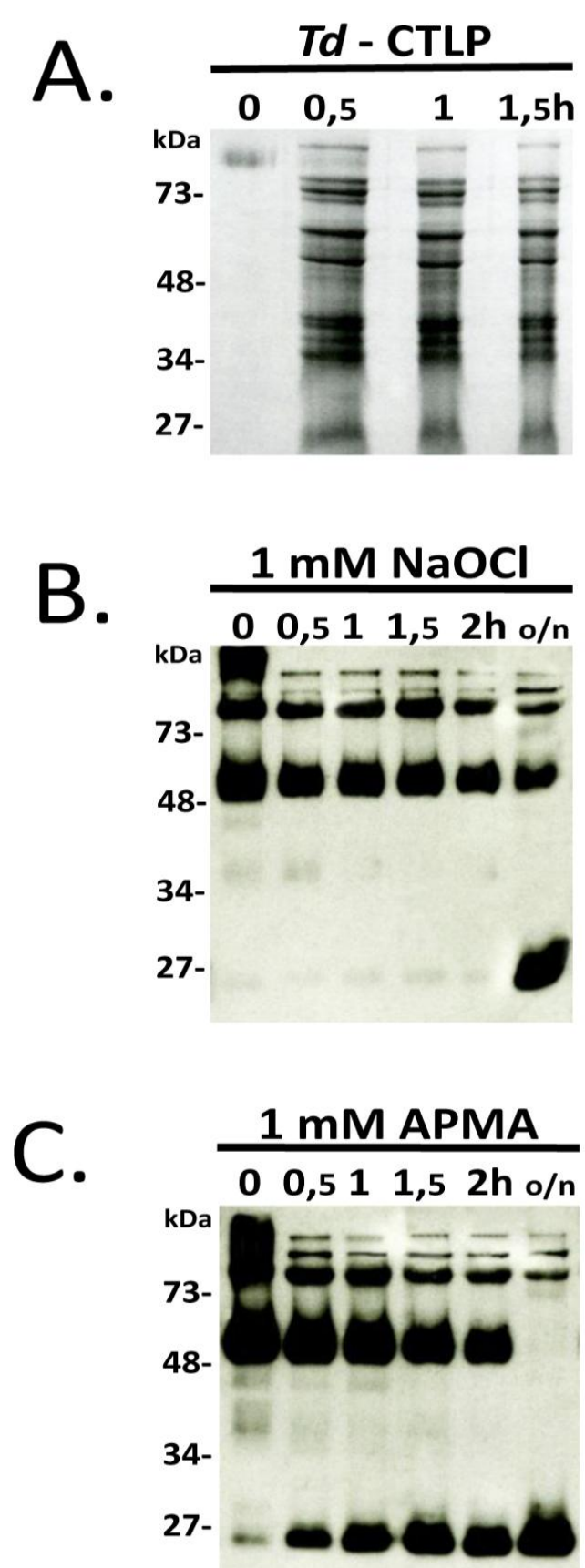


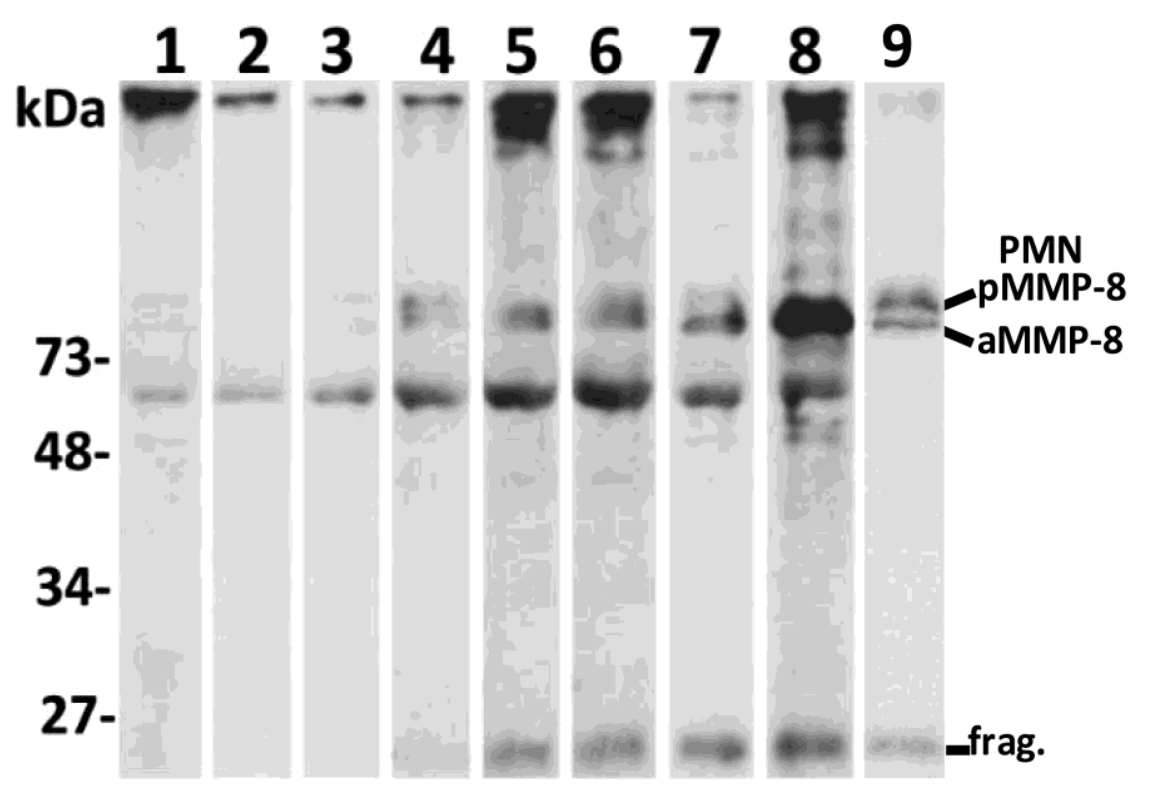

This article is protected by copyright. All rights reserved. 

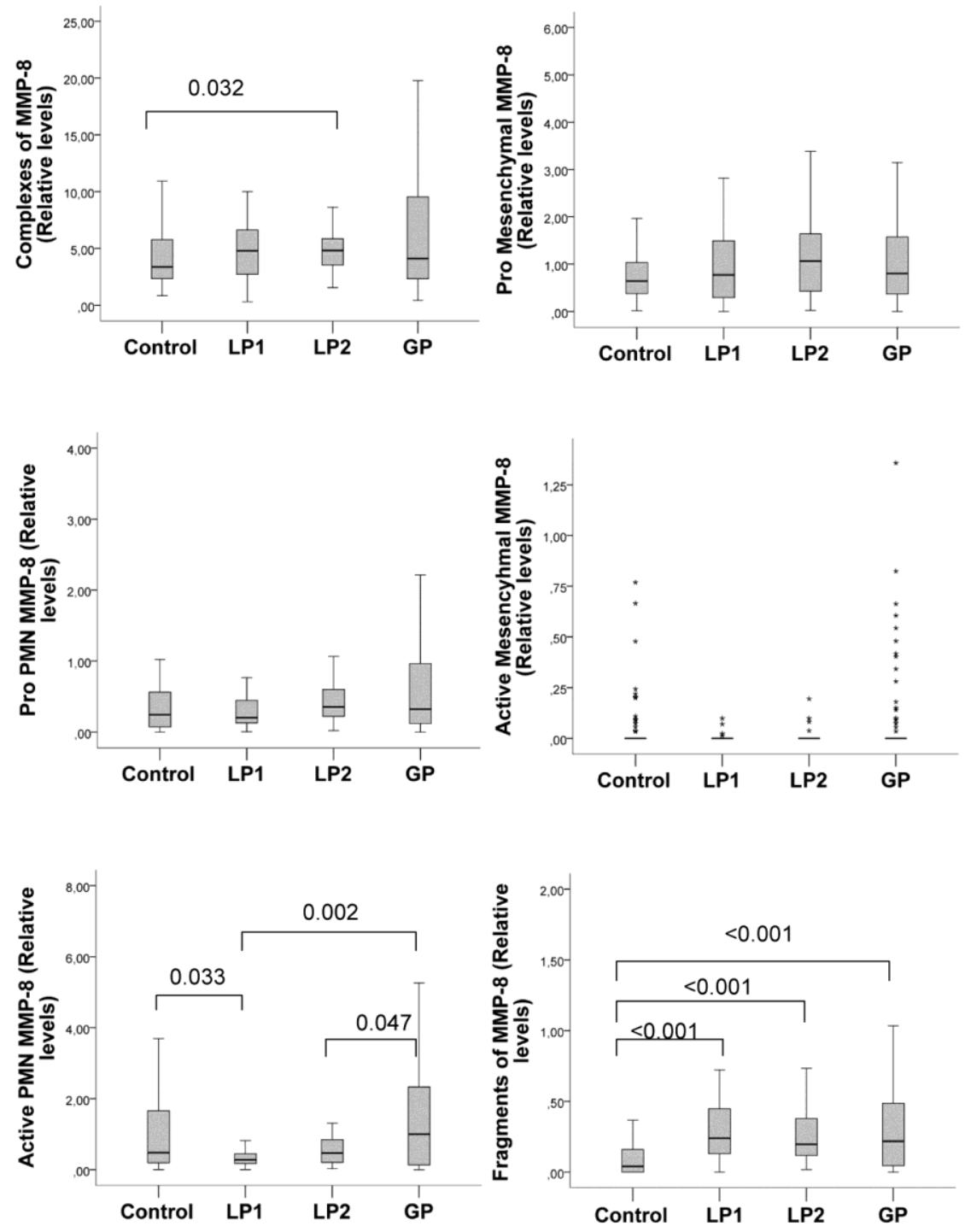

This article is protected by copyright. All rights reserved. 

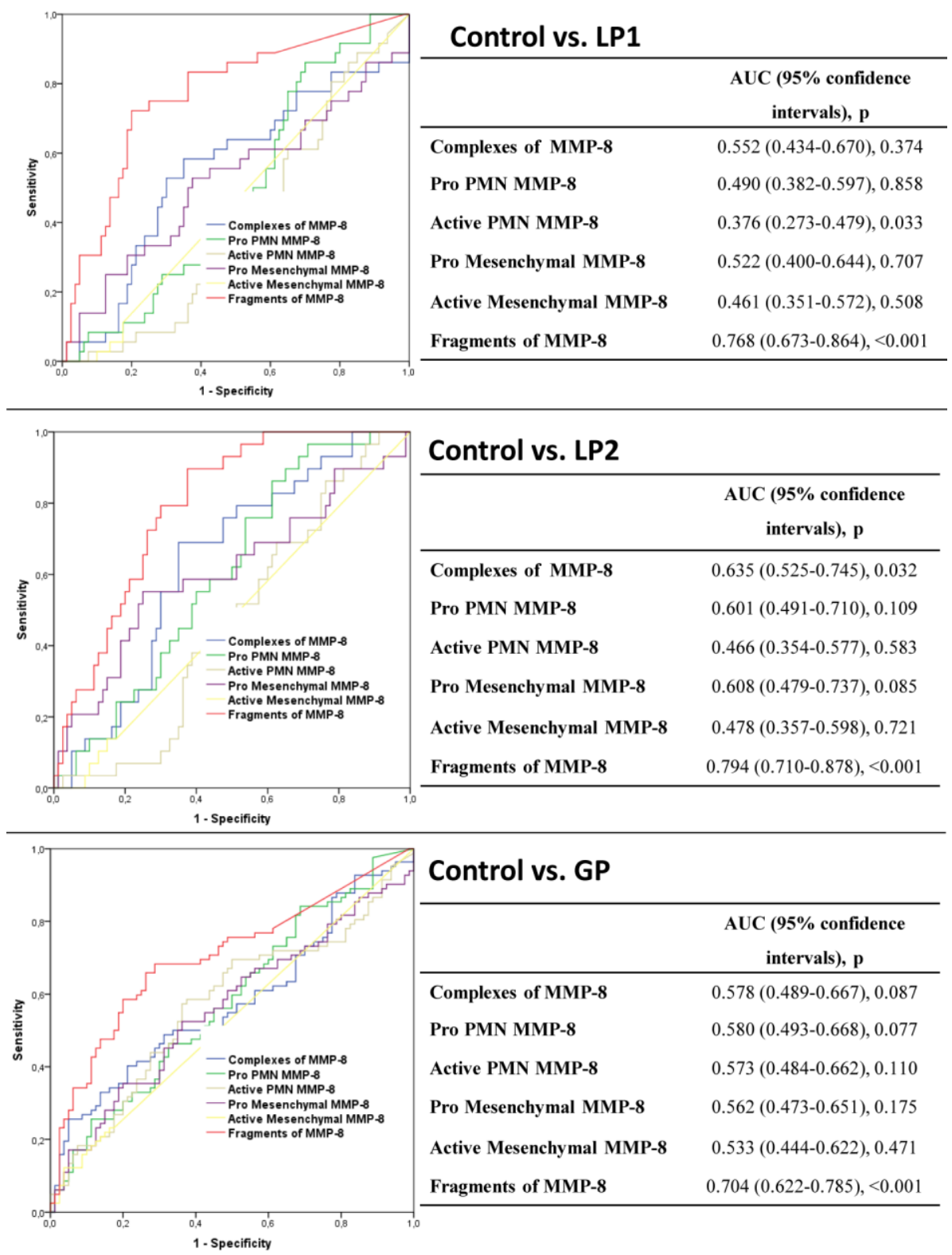

This article is protected by copyright. All rights reserved. 


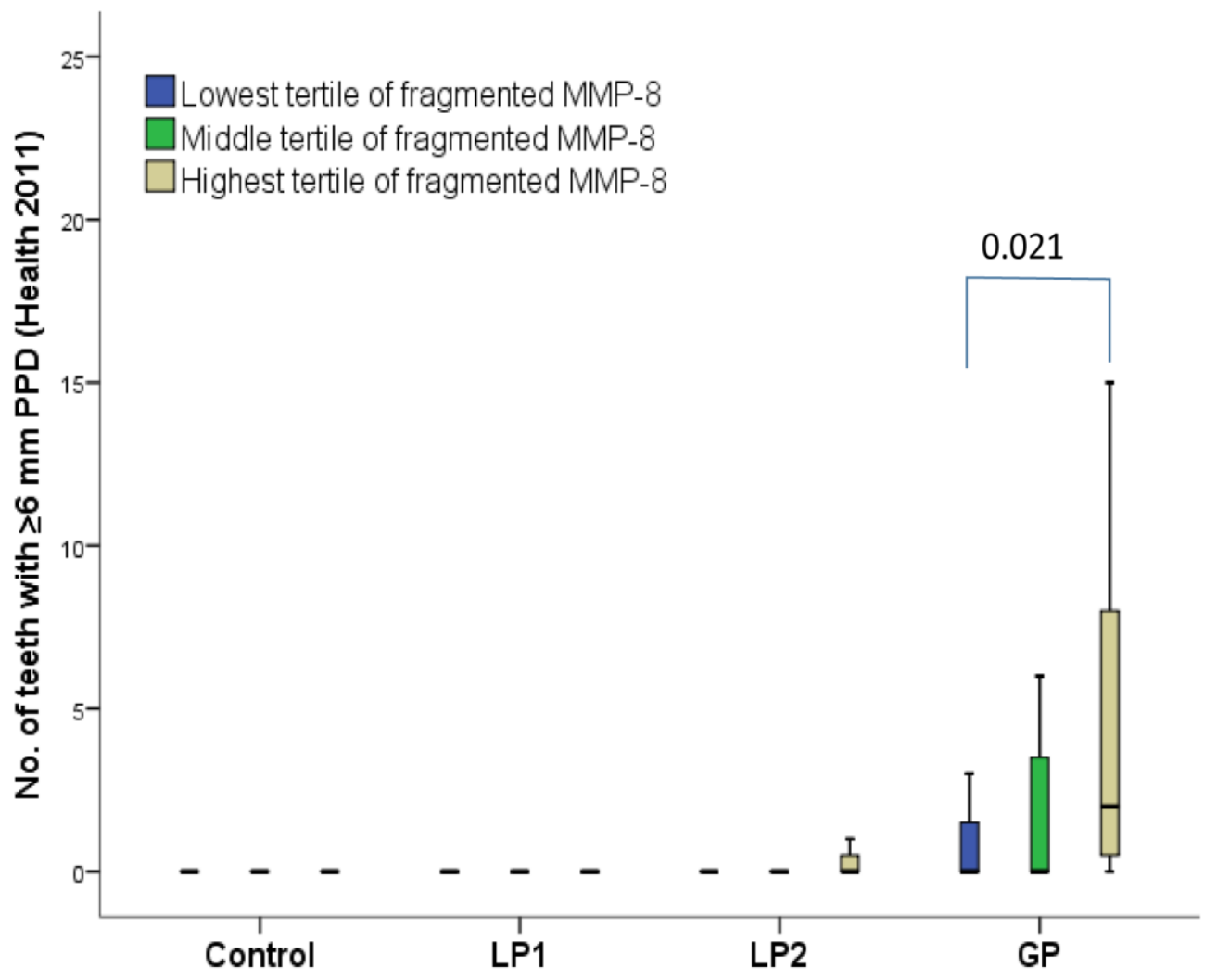

This article is protected by copyright. All rights reserved. 